Check for updates

Cite this: J. Mater. Chem. C, 2020 8,2870

\title{
The optical signatures of molecular-doping induced polarons in poly(3-hexylthiophene-2,5- diyl): individual polymer chains versus aggregates $\dagger$
}

\author{
Ahmed E. Mansour, (D) ab Dominique Lungwitz, (D) a Thorsten Schultz, (D) ab \\ Malavika Arvind, (iD c Ana M. Valencia, (D) a Caterina Cocchi, (iD) a Andreas Opitz, (D) a \\ Dieter Neher (iD ${ }^{c}$ and Norbert Koch (iD *ab
}

\begin{abstract}
Optical absorption spectroscopy is a key method to investigate doped conjugated polymers and to characterize the doping-induced charge carriers, i.e., polarons. For prototypical poly(3-hexylthiophene2,5-diyl) (P3HT), the absorption intensity of molecular dopant induced polarons is widely used to estimate the carrier density and the doping efficiency, i.e., the number of polarons formed per dopant molecule. However, the dependence of the polaron-related absorption features on the structure of doped P3HT, being either aggregates or separated individual chains, is not comprehensively understood in contrast to the optical absorption features of neutral $\mathrm{P} 3 \mathrm{HT}$. In this work, we unambiguously differentiate the optical signatures of polarons on individual P3HT chains and aggregates in solution, notably the latter exhibiting the same shape as aggregates in solid thin films. This is enabled by employing tris(pentafluorophenyl)borane (BCF) as dopant, as this dopant forms only ion pairs with P3HT and no charge transfer complexes, and $\mathrm{BCF}$ and its anion have no absorption in the spectral region of P3HT polarons. Polarons on individual chains exhibit absorption peaks at $1.5 \mathrm{eV}$ and $0.6 \mathrm{eV}$, whereas in aggregates the high-energy peak is split into a doublet $1.3 \mathrm{eV}$ and $1.65 \mathrm{eV}$, and the low-energy peak is shifted below $0.5 \mathrm{eV}$. The dependence of the fraction of solvated individual chains versus aggregates on absolute solution concentration, dopant concentration, and temperature is elucidated, and we find that aggregates predominate in solution under commonly used processing conditions. Aggregates in BCF-doped P3HT solution can be effectively removed upon simple filtering. From varying the filter pore size (down to $200 \mathrm{~nm}$ ) and thin film morphology characterization with scanning force microscopy we reveal the aggregates' size dependence on solution absolute concentration and dopant concentration. Furthermore, X-ray photoelectron spectroscopy shows that the dopant loading in aggregates is higher than for individual P3HT chains. The results of this study help understanding the impact of solution preaggregation on thin film properties of molecularly doped $\mathrm{P} 3 \mathrm{HT}$, and highlight the importance of considering such aggregation for other doped conjugated polymers in general.
\end{abstract}

Received 27th November 2019, Accepted 20th January 2020

DOI: $10.1039 / c 9 t c 06509 a$

rsc.li/materials-c

\section{Introduction}

Fundamental and applied research on organic semiconductors continually grows, high-performance photovoltaic devices and field effect transistors have been demonstrated, and organic light emitting diodes have already entered the market. Organic semiconductors are considered a promising alternative to

\footnotetext{
${ }^{a}$ Institut für Physik \& IRIS Adlershof, Humboldt-Universität zu Berlin, 12489 Berlin, Germany.E-mail: norbert.koch@physik.hu-berlin.de

${ }^{b}$ Helmholtz-Zentrum Berlin für Materialien und Energie GmbH, 12489 Berlin, Germany

${ }^{c}$ Institut für Physik und Astronomie, Universität Potsdam, 14476 Potsdam, Germany $\dagger$ Electronic supplementary information (ESI) available. See DOI: 10.1039/ c9tc06509a
}

inorganic semiconductors for several applications, due to the chemical versatility, mechanical flexibility, and foremost, solubility of most conjugated polymers and small molecules, which enables low-cost solution processing methods for thin films. ${ }^{1}$ Nevertheless, the charge transport properties of organic semiconductors are often not at par with those of their inorganic counterparts, due to the low intrinsic density of mobile charge carriers and carrier mobility. ${ }^{1}$ As for inorganic semiconductors, doping of organics is the key for tailoring transport properties to the needs of applications. Generally, doping proceeds by incorporating a small amount of dopants in the semiconductor with the aim of creating a larger number of mobile charge carriers via ground state charge transfer. Charge carriers in conjugated polymers are spatially rather confined, forming polarons due to 
local bond length alterations, which are typically spread over four monomer units of the polymer. ${ }^{1,2}$ Polarons feature electronic states within the energy gap of the pristine material, ${ }^{3-5}$ and result in additional optical transitions. These can readily be observed in the absorption spectra of doped polymers and thus provide means for characterizing the type of charge carriers, their density, as well as the fraction of dopants that have undergone charge transfer. ${ }^{3,6-8}$ In a one-dimensional polymer chain model, distinct absorption features comprise two peaks for the case of positive charge carriers (positive) polarons due to: (P1) an electronic transitions from the highest occupied electronic energy level to the lower energy level of the polaron in the gap, and (P2) a transition from the highest occupied level to the higher energy level of the polaron. ${ }^{5}$

A primary prototype conjugated polymer, particularly for studies relating to charge carriers and doping, is poly(3-hexylthiophene-2,5-diyl) (P3HT). For example, charge carrier induced optical absorption of the regioregular variant of P3HT (re-P3HT) in thin films, where most polymer chains are ordered in aggregates, was investigated by photo-induced absorption ${ }^{9}$ and charge modulation spectroscopy. ${ }^{10,11}$ In these experiments, notably without the presence of dopants to induce polarons, two distinct absorption features for the $\mathrm{P} 2$ transition at $1.3 \mathrm{eV}$ and $1.7 \mathrm{eV}$ were found. In contrast, the P2 transition for individual chains of re-P3HT encapsulated in a polystyrene matrix exhibited only a single absorption feature at $1.35 \mathrm{eV} .^{9}$ Similarly, for thin film samples of regiorandom P3HT (ra-P3HT), in which coupling among the chains is weak due to the non-planarity and twisting of the thiophene units along the chain, the absorption feature P2 showed a single peak at $1.5 \mathrm{eV} .{ }^{9}$ These apparent differences in the characteristics of the absorption feature $\mathrm{P} 2$ were attributed to differences in the interaction of the polarons with polymer chains in ordered aggregates versus individual chains. ${ }^{9,10,12}$ Furthermore, molecular-scale order of re-P3HT in solution can be controlled by solubility, via choice of solvents or thermal treatment, ${ }^{1}$ i.e., either ordered aggregates or solvated individual chains can (co)exist in solution. It has been shown that the presence of ordered aggregates already in solution significantly influences the properties of the resulting thin films. ${ }^{13}$

Consequently, implications of the presence of aggregates in solution of P3HT mixed with a dopant in solution were also investigated. ${ }^{14-17}$ The abundance of re-P3HT aggregates in solution was reported to increase upon addition of molecular p-type dopants, with 2,3,5,6-tetrafluoro-7,7,8,8-tetracyanoquinodimethane $\left(\mathrm{F}_{4} \mathrm{TCNQ}\right)$ being the one most frequently used, and was ascribed to decreased solubility of the corresponding ionized species after ion pair (IPA) formation (doping involving integer electron transfer) ${ }^{18,19}$ It was shown that aggregation occurs more readily for solution-mixed doping as compared to sequential doping of re-P3HT thin films. ${ }^{20}$ Doping efficiency (fraction of dopants that become ionized), mechanism, and kinetics of solution-mixed doped P3HT are influenced by the regioregularity and molecular-scale order of P3HT in solution before doping, and accordingly, on the relative presence of ordered aggregates and solvated individual chains. For example, Gao et al. showed a distinct difference in the doping efficiency of re- and ra-P3HT, where re-P3HT exhibited higher doping efficiency due to its ability to form ordered aggregates of strongly interacting planar chains that are supposedly supporting delocalization of the generated polarons. In contrast, ra-P3HT does not form ordered aggregates and delocalization is inhibited, resulting in charge transfer complex (CPX) formation instead of IPA, with much lower doping efficiency. ${ }^{14,15}$ Jacobs et al. have recently shown that modifying the crystalline structure of $\mathrm{F}_{4} \mathrm{TCNQ}$ doped re-P3HT thin films can change the doping mechanism from IPA to CPX formation. ${ }^{16}$ Furthermore, the doping process was shown to proceed at different rates for fully solvated individual chains and ordered aggregates of P3HT in solution, with the later exhibiting faster reaction kinetics. ${ }^{17}$

Despite the obvious importance of the molecular-scale order of re-P3HT chains on the doping process, the optical absorption signatures of polarons in the two most abundant environments, i.e., (i) individual chains and (ii) ordered aggregates, are not yet comprehensively clarified for molecularly doped re-P3HT in solution. The absorption spectra of the polaron in molecularly doped re-P3HT thin films found in literature typically show two features for the $\mathrm{P} 2$ transition, i.e., $\mathrm{P} 2_{\mathrm{a}}$ at $\sim 1.3 \mathrm{eV}$ and $\mathrm{P} 2_{\mathrm{b}}$ at $\sim 1.65 \mathrm{eV}$, instead of only one. However, the origin for this has not been investigated in detail, and they have been only referred to as the polaron related transition P2, and in some cases they were attributed to transitions of the bipolaron. ${ }^{8,21,22}$ Perhaps one reason for the present ambiguity is that the commonly used dopant $F_{4}$ TCNQ has its anion absorption peaks in the same spectral region as the polarons of $\mathrm{P} 3 \mathrm{HT}^{7,23,24} \mathrm{~F}_{4} \mathrm{TCNQ}$ anions show two absorption peaks $(1.45 \mathrm{eV} \text { and } 1.6 \mathrm{eV})^{24}$ that are imposed on the polaron related absorption, and thus obstruct a direct observation of its shape. ${ }^{21,22}$

In this contribution, we clearly differentiate the optical absorption signatures of P3HT polarons in doped ordered aggregates on the one hand, and doped individual chains on the other hand, in solution and solid thin films, for P3HT solution-doped with the Lewis acid tris(pentafluorophenyl)borane (BCF) (chemical structures of BCF and re-P3HT are shown in Fig. S1, ESI $\dagger$ ). We have chosen BCF as the dopant because the absorption of neutral BCF $(\sim 4.1 \mathrm{eV})^{25}$ and its anion $(\sim 2.1 \mathrm{eV})^{26}$ after charge transfer do not interfere with the spectral absorption region of polarons in P3HT below $2.0 \mathrm{eV}^{7}$ Furthermore, BCF dopes P3HT exclusively via IPA formation so that P3HT polarons only can be observed, since indications for CPX formation for this material pair have not been reported to date. It should be pointed out that direct IPA formation based on the frontier energy levels of P3HT and BCF can be ruled out, since the electron affinity of BCF is by far insufficient for electron transfer from the valence band top of P3HT (see energy levels of BCF with thiophene oligomers obtained from density functional theory calculations in Fig. S2, ESI $\dagger$ ). As shown in Fig. S2a (ESI $\dagger$ ), there is no hybridization between the molecular orbitals of BCF and thiophene oligomers. This is also confirmed by the corresponding partial charge analysis (see ESI $\uparrow$ ). Rather, it was suggested that doping proceeds via initial generation of a water-BCF complex with substantial Brønsted acidity, followed by protonation of a polymer segment. Subsequently, electron 
transfer from a neutral chain segment to the protonated one (positively charged) occurs, leaving a positive polaron on the former neutral segment. ${ }^{27}$ Here, we show that doped re-P3HT aggregates exhibit a double-peak structure of the P2 transition, which is virtually identical in solution and in thin films. In contrast, the polaron P2 transition for doped individual chains in solution shows a single-peak feature. For solution preparation conditions commonly used in literature, doped ordered aggregates dominate, but they can be largely removed via filtration. Increasing dopant ratio and absolute solution concentration result in increased aggregate size. The yield of IPA formation for each of the two structures is quantified, implying that doped ordered aggregates have higher dopant loading as compared to individual chains.

\section{Experimental methods}

\section{Materials, doping and filtration}

Re-P3HT $\left(M_{\mathrm{w}}=60.2 \mathrm{~kg} \mathrm{~mol}^{-1}\right.$, regioregularity of 97.6\%) was purchased from Merck KGaA (product number: Lisicon ${ }^{\mathbb{R}}$ SP001) and used for all experiments except for the temperature dependent UV-Vis measurements, re-P3HT (RMI-001EE), $\left(M_{\mathrm{w}}=36 \mathrm{~kg} \mathrm{~mol}^{-1}\right.$ and $96 \%$ regioregularity) was obtained from Rieke Metals. Ra-P3HT was purchased from Sigma-Aldrich (Product number: 510823), and BCF was obtained from TCI Deutschland GmbH (product number: T2313). Anhydrous (99.8\%) chlorobenzene (284513 Sigma-Aldrich) was used as a solvent for both the host material (P3HT) and the dopant (BCF). All solutions were prepared in a controlled environment inside a nitrogen glove box $\left(\mathrm{O}_{2}\right.$ and $\left.\mathrm{H}_{2} \mathrm{O}<0.1 \mathrm{ppm}\right)$.

Doping of P3HT was performed by solution mixing, where specific volumes of BCF solution and P3HT solution (BCF added to $\mathrm{P} 3 \mathrm{HT}$ ) were used to produce a certain dopant ratio. The dopant ratio $(\mathrm{A}: \mathrm{B})$ in this work is defined as the ratio of BCF molecules (A) to monomer units of P3HT (B). The absolute concentration of the doped suspension was controlled by controlling the respective concentration of the $\mathrm{P} 3 \mathrm{HT}$ and $\mathrm{BCF}$ solutions before mixing. The mixture was stirred for three days prior to any characterization or film preparation. Thin films were prepared by spin-coating at $700 \mathrm{rpm}$ for 60 seconds, on either solvent cleaned ITO (for XPS and SPM) or glass substrate (for UV-Vis-NIR).

Filtering of suspensions was carried out using polytetrafluoroethylene (PTFE) membranes $(\varnothing 15 \mathrm{~mm})$ from Rotilabo ${ }^{\circledR}$ with a pore size of either $200 \mathrm{~nm}$ (KC94.1) or $450 \mathrm{~nm}$ (KC95.1).

\section{Characterization}

PerkinElmers Lambda950 UV-Vis-NIR spectrophotometer was used to collect the optical absorption spectra in dual beam mode. The spectra were corrected with $100 \%$ transmission. Data were measured with respect to the solvent (in the case of measurements on solution) or the glass substrate (in the case of thin-films measurement). All solution measurements were collected without exposure to air. P3HT solution in dichloromethane (Fig. 1a) was heated in a sealed cuvette using a hot plate operating at $80{ }^{\circ} \mathrm{C}$.
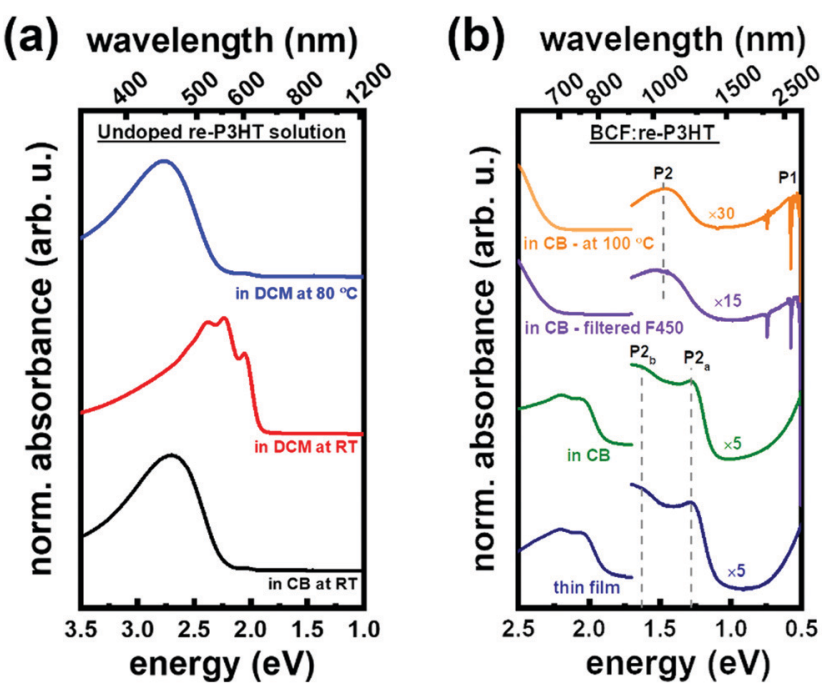

Fig. 1 Optical absorption spectra of ordered aggregates and individual chains of re-P3HT. (a) undoped solutions in $\mathrm{CB}$ at room temperature resembling individual chains (black), DCM at room temperature resembling ordered aggregates (red), and $\mathrm{DCM}$ at $80{ }^{\circ} \mathrm{C}$ (blue) also resembling individual chains. (b) BCF:re-P3HT (doped) highlighting the shape-change of the polaron absorption feature in ordered aggregates in thin films (navy) and solution in $\mathrm{CB}$ (green), versus for individual chains in filtered solution (F450) in CB (purple) and non-filtered CB solution $\left(2 \mathrm{mg} \mathrm{ml}^{-1}\right)$ at $100{ }^{\circ} \mathrm{C}$.

Temperature dependent UV-Vis-NIR measurements of P3HT solution in chlorobenzene (Fig. 1b and Fig. S3, ESI $\dagger$ ) were carried out in ambient air, using a Varian Cary 5000 spectrophotometer fitted with a temperature controlled cuvette holder TLC 50 by Quantum Northwest. The solutions were contained in precision quartz cuvettes of $1 \mathrm{~mm}$ path length. The sample was allowed to equilibrate at each temperature for $c a .10$ minutes before starting the measurement. The total time spent at each temperature was approximately 18 minutes.

Scanning probe microscopy was carried out using a Bruker Dimension Icon system. Surface topography was measured in PeakForce-tapping mode using the Scan Assist module, using ScanAsyst-Air cantilevers. Surface potential was measured using the PeakForce-KPFM mode (frequency modulated) a lift height of $60 \mathrm{~nm}$. SCM-PIT cantilevers were used in KPFM measurements. HOPG was used as a reference to estimate the work function of the tips.

X-ray photoelectron spectroscopy (XPS) was conducted in UHV ( $10^{-9}$ mbar $)$, using a JEOL JPS-9030 system with monochromatic $\mathrm{Al} \mathrm{K} \alpha$ radiation (1486.6 eV). S 2p high-resolution spectra were recorded using a pass energy of $10 \mathrm{eV}$.

\section{Results and discussion}

At first, we compare the optical absorption of ordered aggregates and solvated individual chains in undoped re-P3HT in solution, to set the stage for the subsequent doping studies. As mentioned in the introduction, aggregation of re-P3HT in solution depends on the choice of solvent and/or temperature. Accordingly, re-P3HT forms ordered aggregates in poor solvents, 
such as dichloromethane (DCM) at room temperature, ${ }^{28}$ while it is well solvated into individual chains in chlorobenzene (CB). ${ }^{29}$ Fig. 1a shows the corresponding differences in the absorption spectra of neutral re-P3HT. The absorption of neutral individual re-P3HT chains exhibits a broad feature centered at $\sim 2.7 \mathrm{eV}$, as seen for re-P3HT in $\mathrm{CB}$ at room temperature. In contrast, the absorption spectrum of ordered aggregates in DCM shows wellresolved peaks at $2.0 \mathrm{eV}, 2.2 \mathrm{eV}$, and $2.4 \mathrm{eV}$ in addition to a tail towards higher energy. The red shift of the absorption peak is ascribed to more exciton delocalization that accompanies planarization of $\mathrm{P} 3 \mathrm{HT}$ chains once formed into ordered aggregates, which results in decreasing its energy. ${ }^{19,30-32}$ The absorption fine structure results from the coupling between the electronic and vibrational transitions in crystalline P3HT, as well as the interchain coupling. ${ }^{1,30}$ Increasing the temperature of re-P3HT solution in poor solvents increases solubility, and the re-P3HT aggregates transform to individual chains. This can be seen in the spectrum of re-P3HT in DCM measured at $80{ }^{\circ} \mathrm{C}$ in Fig. 1a, which is identical to that of re-P3HT in $\mathrm{CB}$ at room temperature, (except for the solvent-related shifts in energy position) and no fine structure is observed due to inhomogeneous broadening, ${ }^{30}$ confirming the presence of fully solvated re-P3HT chains.

Having re-iterated the key differences in the optical signatures of neutral re-P3HT in aggregates and in solvated individual chains, we compare the absorption spectra of doped re-P3HT in these two structures. Fig. 1b shows the absorption spectra of $\mathrm{BCF}$ : re-P3HT at a nominal dopant ratio of $1: 4$ (defined as the number of BCF molecules per number of P3HT monomers) in thin film and in CB solution. For both spectra, the region above $2.0 \mathrm{eV}$ shows the absorption fine structure of ordered aggregates of re-P3HT as seen in Fig. 1a. Re-P3HT thin films have been reported to comprise aggregates in the form of relatively large crystalline domains $^{33,34}$ formed during the spin-coating process. ${ }^{13}$ Thus the similarity in the optical absorption of the neutral re-P3HT (thin films and solution) indicates that similar neutral ordered aggregates exist in BCF-doped re-P3HT solution as well.

Below $2.0 \mathrm{eV}$, the absorption of polarons in BCF : re-P3HT $(1: 4)$ thin films and solution comprises the following features: $\mathrm{P} 1$ at or even below $0.5 \mathrm{eV}$, as well as $\mathrm{P} 2_{\mathrm{a}}$ at $1.3 \mathrm{eV}$ and $\mathrm{P} 2_{\mathrm{b}}$ at $1.65 \mathrm{eV}$, as indicated in Fig. 1b. To remove the re-P3HT aggregates from BCF:re-P3HT solution, we passed the solution through a membrane filter having a pore size of $450 \mathrm{~nm}$ (F450) (details in the experimental section). Aggregates larger than $450 \mathrm{~nm}$ are retained in the filter, while allowing the smaller aggregates and individual chains to pass through with the solvent; for the experimental conditions used here the solution comprises predominantly solvated individual chains after filtration. This process is accompanied by a color change of the solution from black for the non-filtered doped solution to orange after filtration as shown in Fig. S1 (ESI $\dagger$ ). The absorption spectrum of the filtered solution (Fig. 1b) resembles that of neutral re-P3HT individual chains above $2.0 \mathrm{eV}$ (compared to the solution spectra of undoped P3HT in Fig. 1a, both in CB at room temperature and in DCM at $80{ }^{\circ} \mathrm{C}$ ). This confirms that the filtration process in our experiment results in the effective removal of the aggregates and that individual re-P3HT chains dominates in the filtered solution. The absorption of polarons on the doped individual chains (filtered solutions) shows a single P2 absorption feature centered at $\sim 1.5 \mathrm{eV}$, and the P1 absorption feature at $\sim 0.5 \mathrm{eV}$. This is distinctly different from the double structured $\mathrm{P} 2$ transition $\left(\mathrm{P} 2_{\mathrm{a}, \mathrm{b}}\right)$ observed for the aggregates in thin films and in solutions, while no changes are observed for the P1 transition (except for a possible slight energy shift) due to the limited spectral range of our setup towards lower energy. To further support the above assignment, we dissolved the aggregates present in BCF:re-P3HT solution by increasing the temperature. Absorption spectra of BCF : re-P3HT $(1: 100)$ in $\mathrm{CB}$ which was heated up to $100{ }^{\circ} \mathrm{C}$ (in steps of $10^{\circ} \mathrm{C}$ ), and subsequently during cooling back to room temperature, are shown in Fig. S3 (ESI $\dagger$ ). The solution was allowed to equilibrate for 10 minutes at the targeted temperature before every measurement step. As the solubility increases with the temperature, the aggregates progressively transform into individual chains of re-P3HT. This is evident by the disappearance of the fine structure in the absorption of the neutral re-P3HT, and the transformation of the polaron absorption from the $\mathrm{P} 22_{\mathrm{a}, \mathrm{b}}$ double-features into the single P2 peak, which prevails at temperatures higher than $70{ }^{\circ} \mathrm{C}$. The latter is virtually identical with the absorption of the filtered doped solution at room temperature. The spectrum of BCF:re-P3HT $(1: 100)$ in CB at $100{ }^{\circ} \mathrm{C}$ is shown in Fig. $1 \mathrm{~b}$ for direct comparison. As the solution cools down to room temperature, the individual chains assembled into the ordered aggregates, and the typical spectrum of ordered aggregates (both neutral and doped) is reproduced (Fig. S3a, ESI $\dagger$ ).

Yet, to further substantiate our assignment, we measured the optical absorption of BCF : ra-P3HT (1:4), where no aggregate formation in solution is expected due to the randomly linked hexyl side chains along the polymer (low percentage of head-to-tail linkage). ${ }^{31,35}$ In Fig. S4a (ESI $\dagger$ ), the optical absorption spectrum of BCF : ra-P3HT $(1: 4)$ in solution shows a single $\mathrm{P} 2$ absorption feature at $1.5 \mathrm{eV}$ in addition to the P1 absorption feature at $0.6 \mathrm{eV}$ in agreement with our observation in the absorption of individual chains of doped re-P3HT. The blue shift of the P1 transition, as compared to re-P3HT, is most likely due to the different electronic structure of these two regioregularities of $\mathrm{P} 3 \mathrm{HT} .{ }^{15}$ Furthermore, filtering the solution of BCF:ra-P3HT did not change the absorption spectrum, as shown in Fig. S4b (ESI $\dagger$ ), i.e., all individual chains (with dopants) could pass through the filter.

The effect of the dopant ratio and absolute solution concentration on aggregates for BCF : re-P3HT solutions is discussed next. Fig. 2a shows the optical absorption spectra of BCF : re-P3HT in CB as a function of dopant ratio, at a constant absolute concentration of $1 \mathrm{mg} \mathrm{ml}{ }^{-1}$. Upon adding BCF solution to re-P3HT solution, an intensity increase of the peaks at $2.0 \mathrm{eV}$ and $2.2 \mathrm{eV}$ (attributed neutral ordered aggregates) is observed, accompanied with a decrease of the peak at $2.7 \mathrm{eV}$ (solvated neutral individual chains). ${ }^{17,30,32}$ Simultaneously, the polaron absorption grows in intensity with increasing dopant ratio, and in all cases it comprises $\mathrm{P} 2 \mathrm{a}(1.3 \mathrm{eV})$ and $\mathrm{P} 2 \mathrm{~b}(1.65 \mathrm{eV})$, as well as $\mathrm{P} 1$ below $0.5 \mathrm{eV}$. These spectral changes can be seen as a color change from clear 
(a)

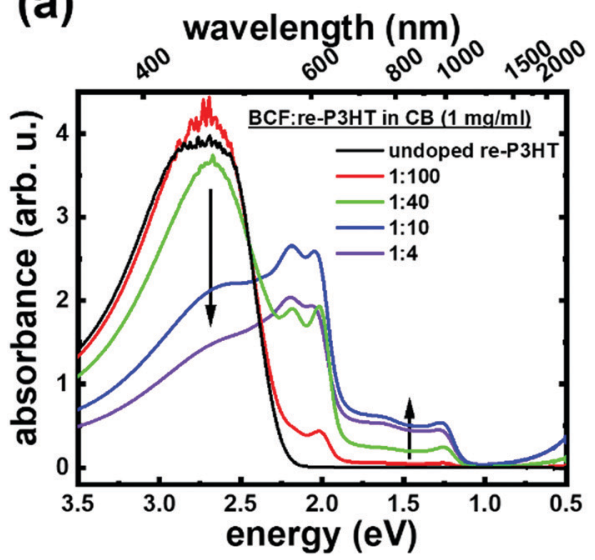

(b)

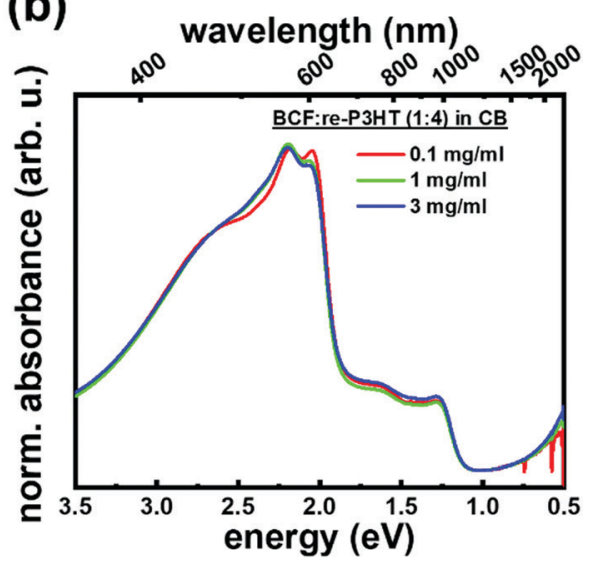

Fig. 2 Optical absorption spectra of $\mathrm{BCF}$ : re-P3HT in $\mathrm{CB}$ at (a) different dopant ratios at an absolute concentration of $1 \mathrm{mg} \mathrm{ml}^{-1}$, and (b) different absolute concentration at a dopant ratio $1: 4$. In (b), all spectra are normalized to the neutral P3HT absorption at $2.7 \mathrm{eV}$.

orange - characteristic of well dissolved re-P3HT - to brown, and eventually black at high dopant ratios (higher than 1:40), as shown in Fig. S1 (ESI $\dagger$ ). These observations indicate that doping-induced aggregation significantly increases the amount of neutral as well as charged aggregates in solution, both increasing with increasing dopant ratio. The simultaneous increase in the absorption of the neutral aggregates $(2.0 \mathrm{eV}$ and $2.2 \mathrm{eV}$ ) and of the doped aggregates (1.3 eV and $1.65 \mathrm{eV})$ indicates that both charged and neutral phases co-exist. Fig. $2 \mathrm{~b}$ shows the optical absorption spectra of BCF : re-P3HT in CB at constant dopant ratio (1:4) as a function of absolute concentration. For all, similar absorption features are observed, with characteristic aggregate features of both neutral and positively charged re-P3HT. Notably, the only slight overall spectral dependence on solution concentration indicates that the relative amounts of ordered aggregates (both neutral and positively charged) compared to neutral solvated chains is constant in this concentration range, and that this ratio is solely determined by the dopant ratio (compare Fig. 2a in the spectral region above $2 \mathrm{eV}$ ).

Next, we investigate the effect of the dopant ratio and absolute concentration on the apparent size of the aggregates in solution. BCF : re-P3HT in CB - prepared with different dopant ratio and absolute solution concentration - was filtered sequentially with two filters of decreasing pore size, namely $450 \mathrm{~nm}$ (F450) and $200 \mathrm{~nm}$ (F200). Accordingly, the aggregates could be classified based on their size into (i) larger than $450 \mathrm{~nm}$, (ii) between $450 \mathrm{~nm}$ and $200 \mathrm{~nm}$ (passing through F450 but retained in F200), and (iii) smaller than $200 \mathrm{~nm}$. Fig. 3a compares the absorption spectra of BCF : re-P3HT 1 : 4 and $3 \mathrm{mg} \mathrm{ml} \mathrm{m}^{-1}$ ) before and after filtration. Passing the solution through F450 results in effective removal of the aggregates as indicated by the disappearance of the peaks at $2.0 \mathrm{eV}$ and $2.2 \mathrm{eV}$, and the change of the polaron absorption features from the two peaks $\mathrm{P} 2_{\mathrm{a}, \mathrm{b}}$ to the single peak $\mathrm{P} 2$. This indicates that in such solutions the aggregates are mostly larger than $450 \mathrm{~nm}$. In contrast, for solutions with 1:10 dopant ratio and $3 \mathrm{mg} \mathrm{ml} \mathrm{m}^{-1}$ concentration, as well as $1: 4$ and $1 \mathrm{mg} \mathrm{ml}^{-1}$, the signatures of the aggregates persist after filtering with F450, yet their intensity is reduced, as seen in Fig. $3 \mathrm{~b}$ and c, respectively. Subsequent filtration with F200 leads to very effective removal of aggregates, as apparent from the disappearance of the neutral aggregates' features and the $\mathrm{P} 2_{\mathrm{a}, \mathrm{b}}$ polaron double peak. This shows that the aggregates in BCF:re-P3HT solution prepared at these conditions are mostly smaller than $450 \mathrm{~nm}$ but larger than $200 \mathrm{~nm}$. For lower dopant ratio at lower concentration,
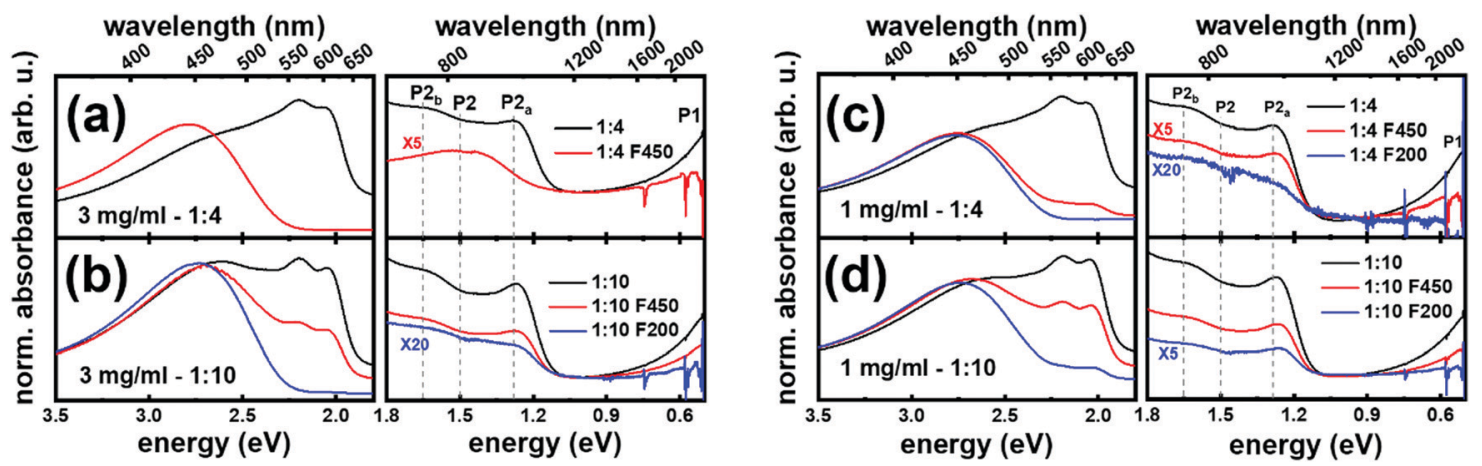

Fig. 3 Changes of optical absorption spectra of BCF : re-P3HT in CB upon filtration for absolute concentration of $3 \mathrm{mg} \mathrm{ml}^{-1}$ and dopant ratios of (a) $1: 4$ and (b) 1:10; and absolute concentration of $1 \mathrm{mg} \mathrm{ml}^{-1}$ and dopant ratios of (c) $1: 4$ and (d) $1: 10$. All spectra are normalized to the absorption at 2.7 eV. 
the absorption features of aggregates persist even after using the F200 filter, as seen in Fig. 3d for the dopant ratio $1: 10$, and in Fig. S5 (ESI $\dagger$ ) for 1:40 and 1:100 at an absolute concentration of $1 \mathrm{mg} \mathrm{ml}^{-1}$. This indicates that a significant fraction of aggregates is smaller than $200 \mathrm{~nm}$. Accordingly, dopant induced aggregates in BCF : re-P3HT solutions increase in size with increasing the dopant ratio and/or the absolute concentration. Furthermore, it was possible to induce aggregation in the filtered solution again, upon adding more dopant (see Fig. S6b, ESI $\dagger$ ). Recalling that aggregation of re-P3HT in solution is driven by lowering the solubility as discussed earlier, we note that the average size of the aggregates increases with both the dopant ratio and absolute concentration, due to the doping induced decrease of solubility as the absolute amount of charged re-P3HT chains/segments increases.

We attempted to dissolve the aggregates by diluting a $\mathrm{BCF}$ : re-P3HT solution (initially $1: 4$ dopant ratio and $3 \mathrm{mg} \mathrm{ml}^{-1}$ concentration) by a factor of 1000 . However, aggregates persisted in solution, as shown in Fig. S7 (ESI $\dagger$ ). The absorption of polarons after dilution still showed the characteristic $\mathrm{P} 2 \mathrm{a}, \mathrm{b}$ features, but with decreased intensity, while the absorption from re-P3HT indicated an increase of neutral individual chains as compared to neutral aggregates. Yet, the presence of the dopants in re-P3HT aggregates enhances aggregate stability in solution. Comparing this to the effect of temperature on BCF:re-P3HT solutions in CB (up to $100{ }^{\circ} \mathrm{C}$ ) presented in Fig. S3a (ESI $\dagger$ ), we recall that polaron absorption changed from the double $\mathrm{P} 2_{\mathrm{a}, \mathrm{b}}$ features to a single $\mathrm{P} 2$ peak already at $70{ }^{\circ} \mathrm{C}$. This was accompanied by a reduction of the neutral re-P3HT aggregate signature, and was ascribed to improved solvation of doped re-P3HT at elevated temperature. ${ }^{8}$ The difference in the observed behavior between increasing the solubility via dilution and heating can be ascribed to the different dependency of solubility on absolute concentration on one hand, or temperature on the other hand. In addition, we speculate that de-doping of the BCF:re-P3HT system at elevated temperature may play a role, by acting as a pathway to dissolve the doped aggregates followed by dopants binding to isolated chains in solution, which can then re-aggregate once the solution cools down to room temperature. Fig. S3b (ESI $\dagger$ ) shows conductivity measurements on BCF : re-P3HT thin films as a function of temperature. Conductivity decreases for all dopant ratio at $80{ }^{\circ} \mathrm{C}$, and is attributed to the de-doping of P3HT at this point. The temperature at which de-doping occurs roughly agrees with the temperature at which the double $\mathrm{P} 2_{\mathrm{a}, \mathrm{b}}$ polaron absorption feature changes to a single P2 peak (Fig. S3a, ESI $\dagger$ ). To assess the impact of aggregates in solution on solution-processed thin films, we compared the morphology obtained from scanning force microscopy of BCF : re-P3HT (1:4) thin films prepared from solutions with different absolute concentration, before and after filtration (the concentration after filtration is certainly lower than that of the pristine solution). The top row of Fig. 4 shows that the aggregates can already be observed in thin films spin coated from solution with the lowest absolute concentration $\left(0.1 \mathrm{mg} \mathrm{ml}^{-1}\right)$. The apparent aggregates have the form of $\sim 2 \mu \mathrm{m}$ long and $\sim 30 \mathrm{~nm}$ wide fibrils. When the absolute concentration of the solution is increased to $1 \mathrm{mg} \mathrm{ml} \mathrm{me}^{-1}$, we observe an increased density of the aggregates (similar size to the previous case), appearing as an interconnected network of the fibrils. Further increasing the absolute concentration to $3 \mathrm{mg} \mathrm{ml} \mathrm{m}^{-1}$ results in a rather corrugated surface, where it is hardly possible to discern individual fibrils. Overall features seem around $\sim 100 \mathrm{~nm}$ wide and they cover the entire surface, which could be understood as many fibrils with arbitrary orientation (now including the third dimension, i.e., film thickness) forming a dense network. The morphology of thin films prepared from filtered solution using F450 is shown in the bottom row of Fig. 4. Thin films prepared from $0.1 \mathrm{mg} \mathrm{ml}^{-1}$ (F450) solution still show some aggregates in the form of fibrils, but also smaller dot-like aggregates, in agreement with our
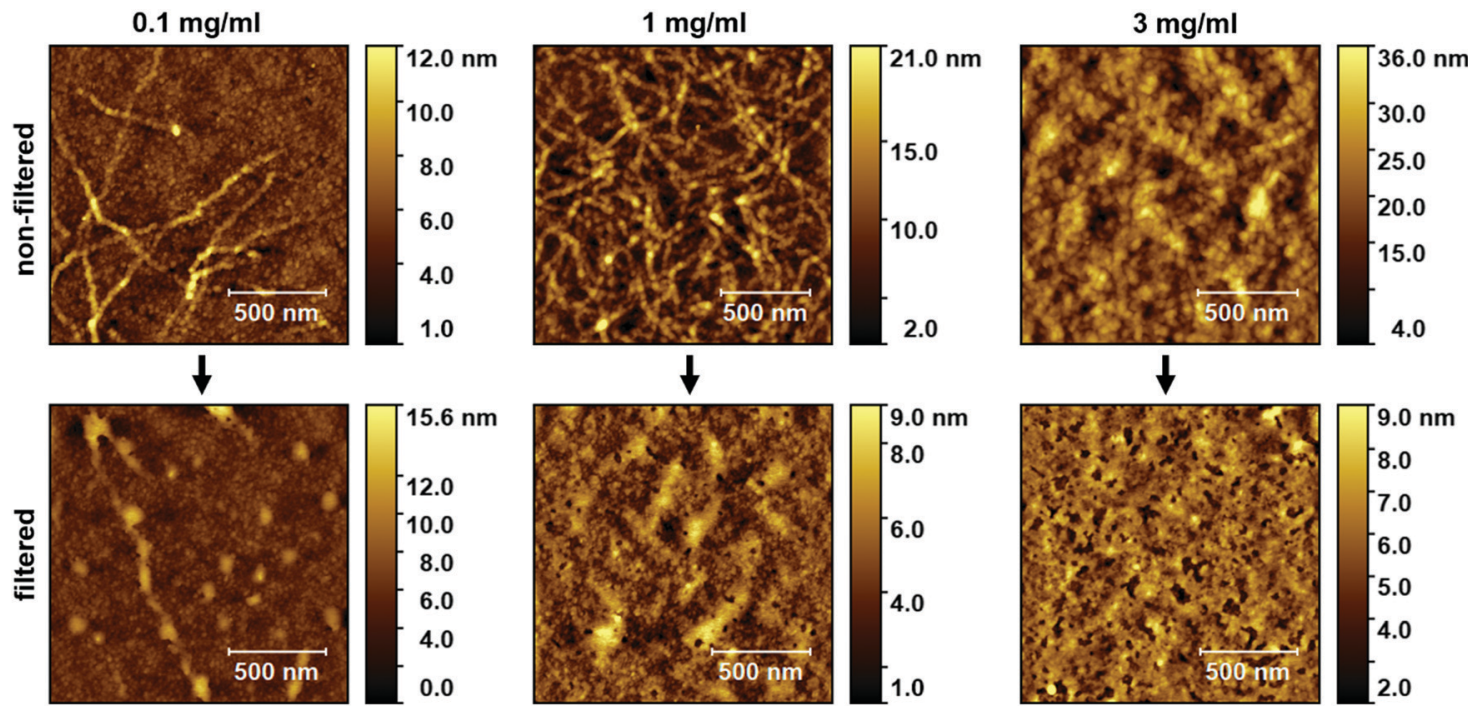

Fig. 4 Scanning force microscopy images of BCF : re-P3HT (1:4) thin films spin coated from as-prepared (non-filtered) solutions with varying absolute concentrations (top row), and after filtration using F450 (bottom row). 
findings from absorption spectra that smaller-sized aggregates could pass through F450 (see Fig. S6a, ESI $\dagger$ ). Thin films prepared from filtered solutions with absolute concentrations of $1 \mathrm{mg} \mathrm{ml}$ and $3 \mathrm{mg} \mathrm{ml}^{-1}$ exhibit a comparably homogenous morphology and smooth surfaces, albeit with some pinholes probably due to the decreased concentration after filtration. Some protruding features can be observed in films prepared from $1 \mathrm{mg} \mathrm{ml} \mathrm{m}^{-1}$ (F450) solution, attributed to possible agglomeration of smaller aggregates that could pass through F450 (Fig. S6a, ESI $\dagger$ ).

The gradual appearance of the aggregates in thin films, forming larger networks and followed by agglomeration with the increase of absolute concentration of the solution, is in line with our findings discussed for Fig. 3 and Fig. S6a (ESI $\dagger$ ), in which we found a direct dependency of the aggregate size on absolute concentration of the BCF:re-P3HT solution. The morphology of thin films prepared from filtered BCF:re-P3HT solutions (bottom row in Fig. 4) reveales that the aggregates observed in thin films prepared from non-filtered solution (top row in Fig. 4) are primarily not due to aggregation during drying of the film formation process, but predominantly from aggregates that already existed in the solution prior to spin coating.

Having established the clear differentiation of aggregates and individual chains in doped re-P3HT from optical absorption spectroscopy, we turn towards the effectiveness of doping for these two different structures, here assessed through X-ray photoelectron spectroscopy (XPS). We define the degree of doping as the ratio of sulfur atoms experiencing the positive charge (polaron) to the total number of sulfur atoms in P3HT. Thin films $(\sim 10 \mathrm{~nm})$ were coated on indium tin oxide (ITO) substrates from non-filtered and filtered solutions (F200). Two different dopant ratios are compared: (i) $1: 4\left(1 \mathrm{mg} \mathrm{ml}^{-1}\right)$ for which filtration results in dominantly doped individual chains in solution as established by optical absorption (Fig. 3c), and (ii) $1: 40\left(1 \mathrm{mg} \mathrm{ml}^{-1}\right)$ in which doped aggregates still dominate the solution after filtration according to the optical absorption spectrum in Fig. S5a (ESI $\dagger$ ). High-resolution scans of $\mathrm{S} 2 \mathrm{p}$ energy region were collected and deconvoluted with two components (each comprising the doublet of $S 2 p_{3 / 2}$ and $S 2 p_{1 / 2}$ ). The neutral sulfur component is centered at $\sim 163.3 \mathrm{eV}\left(\mathrm{S} 2 \mathrm{p}_{3 / 2}\right)$ while the sulfur atoms with positive charge (within a polaron) are shifted by $0.6 \mathrm{eV}$ towards higher binding energy. ${ }^{36,37}$ Details of the peak fitting are given in the ESI, $\dagger$ and the fitting parameters are summarized in Table S1 (ESI $\dagger$ ). Fig. 5 shows the accordingly deconvoluted S 2p spectra along with the computed degree of doping. Thin films coated from non-filtered BCF : re-P3HT (1:4), exhibit a degree of doping of $17 \%$. In contrast, thin films prepared from the filtered solution, which contains mainly doped individual chains, shows a degree of doping of $6 \%(\sim 65 \%$ reduction of positively charged sulfur). Apparently, the degree of doping is significantly higher in ordered aggregates as compared to individual chains of doped re-P3HT, which indicates that aggregates should contribute more doping-induced charge carriers, in agreement with previous art. ${ }^{14}$ For the lower dopant ratio of $1: 40$, thin films prepared from non-filtered and filtered solution show no significant change in the degree of doping, since the aggregates persist after filtration due to their smaller size. However, it is

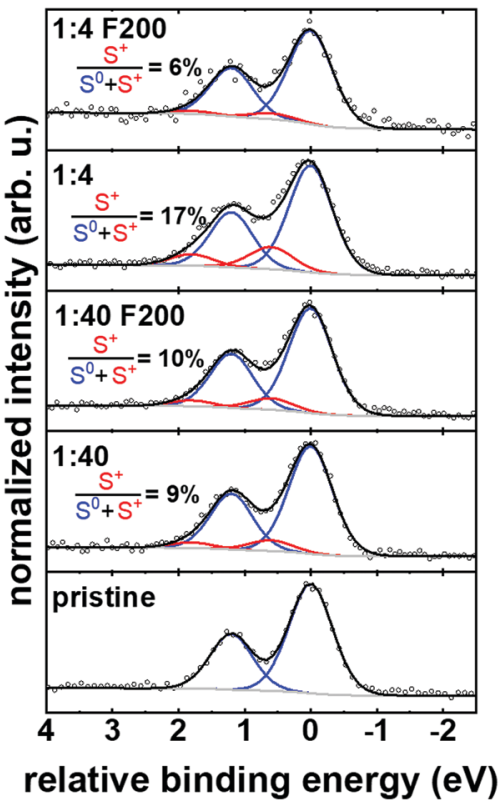

Fig. 5 XPS high-resolution S 2p spectra and the computed degree of doping in BCF : re-P3HT thin films prepared from $1 \mathrm{mg} \mathrm{ml}^{-1}$ solutions at the indicated dopant ratios, before and after filtration using F200. All peak maxima ( $\left(\mathrm{S}_{3 / 2}\right)$ are set to zero for comparison. All films were prepared by spin coating except for 1: 4 F200, which was drop casted, since obtaining films of sufficient coverage was not possible with spin coating.

expected that some of the aggregates are of larger size and thus are removed by F200 as shown in Fig. S5 (ESI $\dagger$ ). Thus, the fact that the degree of doping barely changed upon filtering the $1: 40$ solution indicates that the dopant loading in the aggregates is independent of their size. Furthermore, we observed a lower work function (obtained from the measured contact potential difference by Kelvin-probe force microscopy) for thin films without aggregates compared to those with aggregates (see Fig. S8, ESI $\dagger$ ). This observation is fully in line with the lower degree of doping of individual chains.

Finally, we discuss the possible origin of the double-peak features $\mathrm{P} 2_{\mathrm{a}, \mathrm{b}}$ in doped aggregates of re-P3HT as contrasted to the single absorption feature $\mathrm{P} 2$ found in doped individual chains. The absorption of neutral individual chains of re-P3HT features a broad peak at $\sim 2.7 \mathrm{eV}$, stemming from the first $\pi \rightarrow \pi^{*}$ electronic transition, coupled with vibrational excitations, which remain spectrally unresolved due to conformational disorder. Similarly, we observed the absorption of the polarons on doped individual chains to comprise a single broad feature at $\sim 1.5 \mathrm{eV}$, originating from the electronic transition P2. In contrast, the absorption of neutral aggregates of re-P3HT shows a fine structure of well-resolved peaks, at $2.0 \mathrm{eV}, 2.2 \mathrm{eV}$, and $2.4 \mathrm{eV}$, resulting from the fundamental electronic transition in coupled chains and coupled to vibrations. Doped aggregates exhibit two absorption features $\mathrm{P} 2_{\mathrm{a}}(1.3 \mathrm{eV})$ and $\mathrm{P} 2_{\mathrm{b}}(1.65 \mathrm{eV})$, which plausibly could also involve inter-chain coupling and/or coupling to a dominant vibration. However, we observe that the separation between $\mathrm{P} 2_{\mathrm{a}}$ and $\mathrm{P} 2_{\mathrm{b}}(\sim 350 \mathrm{meV})$ is rather large compared to the aromatic vibrations of thiophene ${ }^{38}$ 
$(<200 \mathrm{meV})$, making it unlikely that this splitting is due to vibrational progression only. Additionally, we exclude the possibility of assigning these features to electronic transitions related to the formation of bipolarons. Our XPS quantitative analysis, indicates that for films prepared from non-filtered doped BCF : P3HT solution (1:4), one polaron exists per ca. 24 monomer units (Table S2, ESI $\dagger$ ). At such low density of polarons, the formation of stable bipolarons is unlikely since it requires two polarons to form on a chain shorter than seven monomer units. ${ }^{6,39}$ Furthermore, bipolarons are accompanied with electronic states deeper in the band gap of P3HT as compared to polarons, due to larger geometric relaxation in the polymer chain. ${ }^{4}$ Accordingly, optical absorption peaks due to bipolarons are expected to be red shifted as compared to those of polaron related absorption. Indeed, this has been experimentally observed in chemically ${ }^{40}$ and electrochemically ${ }^{6}$ doped P3HT. In addition, it was confirmed that BCF doped P3HT forms mainly polarons using electron paramagnetic resonance spectroscopy, where the optical absorption spectra have shown both of the two absorption features, $\mathrm{P} 2 \mathrm{a}$ and $\mathrm{P} 22_{\mathrm{b}}{ }^{8}$ We may thus consider Davydov splitting as the origin of the observed splitting. Here, the interaction of adjacent polymer chains in ordered crystals where the unit cell is comprised of dimers, could indeed result in a double-peak in the absorption spectrum. Previous studies on the crystalline structure of undoped P3HT revealed unit cells comprised of two P3HT chains. ${ }^{41-43}$ Structural studies on F4TCNQ doped re-P3HT have shown that the $\pi-\pi$ packing distance decreases in a mixed crystalline phase. ${ }^{16,44}$ Jacobs et al. recently reported that the lamellar packing distance also decreases along with the $\pi-\pi$ packing distance upon doping under certain processing conditions, in unit cells containing two doped P3HT chains. These structural studies, despite being done on different doped systems, support the possibility of Davydov splitting in the ordered aggregates of doped re-P3HT. Calculations of the Davydov splitting in oligothiophene molecular crystals were reported to be in the range of $0.3-0.6 \mathrm{eV}$, depending on the number of the monomers, as well as the size of the aggregates. ${ }^{45-47}$ Accordingly, we suggest that the absorption features $\mathrm{P} 2_{\mathrm{a}}$ and $\mathrm{P} 2_{\mathrm{b}}$ in the aggregates could originate from Davydov splitting of highenergy polaron transition $\mathrm{P} 2$ in re-P3HT.

\section{Conclusion}

In conclusion, we reveal that doping of re-P3HT with BCF in solution results in the formation of a suspension, in which doped ordered aggregates and doped individual chains coexist, but aggregates predominate at commonly employed processing conditions. The optical signatures of polarons in these two structures were identified by physical separation using filtration. Polarons in aggregates of re-P3HT, both in thin films and solution, exhibit three absorption peaks, namely, $\mathrm{P} 2_{\mathrm{a}}$ at $1.3 \mathrm{eV}$ and $\mathrm{P} 2_{\mathrm{b}}$ at $1.65 \mathrm{eV}$, in addition to $\mathrm{P} 1$ below $0.5 \mathrm{eV}$. In contrast, polarons on individual P3HT chains show only two absorption peaks, i.e., $\mathrm{P} 2$ at $1.5 \mathrm{eV}$ and $\mathrm{P} 1$ at $c a$. $0.5 \mathrm{eV}$. The presence of dopants enhances the stability of the aggregates in solution, which increase in size with both dopant concentration and the absolute solution concentration. Solution heating dissolves aggregates, but they re-form upon cooling to room temperature. Ordered aggregates are shown to have higher affinity to doping as compared to individual chains. The presented study provides a reliable basis for spectroscopic characterization of the structure of doped P3HT in solution, a fundamental understanding of the dopant induced aggregation process, and the influence of the solution preparation conditions. This insight is essential for controlling the size of aggregates along with the doping efficiency in thin films as used in devices. Understanding solution aggregation of doped conjugated polymers and its impact on thin film properties is thus generally essential, when optimized performance and highest reproducibility are aimed at.

\section{Conflicts of interest}

There are no conflicts to declare.

\section{Acknowledgements}

This work was funded by the German Research Foundation (DFG) through the project "FoMEDOS" (project number 286798544). A. M. V. and C. C. acknowledge the North-German Supercomputing Alliance (HLRN) for computational resources through the project bep00076.

\section{References}

1 A. Köhler and H. Bässler, Electronic Processes in Organic Semiconductors, Wiley-VCH Verlag GmbH \& Co. KgaA, Weinheim, Germany, 2015.

2 P. Pingel, L. Zhu, K. S. Park, J. O. Vogel, S. Janietz, E. G. Kim, J. P. Rabe, J. L. Brédas and N. Koch, Charge-Transfer Localization in Molecularly Doped Thiophene-Based Donor Polymers, J. Phys. Chem. Lett., 2010, 1(13), 2037-2041.

3 T. C. Chung, J. H. Kaufman, A. J. Heeger and F. Wudl, Charge Storage in Doped Poly(Thiophene): Optical and Electrochemical Studies, Phys. Rev. B, 1984, 30(2), 702-710.

4 J. L. Bredas and G. B. Street, Polarons, Bipolarons, and Solitons in Conducting Polymers, Acc. Chem. Res., 1985, 18(10), 309-315.

5 G. Heimel, The Optical Signature of Charges in Conjugated Polymers, ACS Cent. Sci., 2016, 2(5), 309-315.

6 C. Enengl, S. Enengl, S. Pluczyk, M. Havlicek, M. Lapkowski, H. Neugebauer and E. Ehrenfreund, Doping-Induced Absorption Bands in P3HT:Polarons and Bipolarons, ChemPhysChem, 2016, 17(23), 3836-3844.

7 P. Pingel and D. Neher, Comprehensive Picture of P-Type Doping of P3HT with the Molecular Acceptor F4TCNQ, Phys. Rev. B, 2013, 87(11), 115209.

8 P. Pingel, M. Arvind, L. Kölln, R. Steyrleuthner, F. Kraffert, J. Behrends, S. Janietz and D. Neher, P-Type Doping of Poly(3-Hexylthiophene) with the Strong Lewis Acid Tris(Pentafluorophenyl)Borane, Adv. Electron. Mater., 2016, 2(10), 1600204 
9 R. Oesterbacka, C. P. An, X. M. Jiang and Z. V. Vardeny, Two-Dimensional Electronic Excitations in Self-Assembled Conjugated Polymer Nanocrystals, Science, 2000, 287(5454), 839-842.

10 H. Sirringhaus, P. J. Brown, R. H. Friend, M. M. Nielsen, K. Bechgaard, B. M. W. Langeveld-Voss, A. J. H. Spiering, R. A. J. Janssen, E. W. Meijer, P. Herwig and D. M. de Leeuw, Two-Dimensional Charge Transport in Self-Organized, HighMobility Conjugated Polymers, Nature, 1999, 401(6754), 685-688.

11 P. J. Brown, H. Sirringhaus, M. Harrison, M. Shkunov and R. H. Friend, Optical Spectroscopy of Field-Induced Charge in Self-Organized High Mobility Poly(3-Hexylthiophene), Phys. Rev. B, 2001, 63(12), 125204.

12 D. Beljonne, J. Cornil, H. Sirringhaus, P. J. Brown, M. Shkunov, R. H. Friend and J. L. Brédas, Optical Signature of Delocalized Polarons in Conjugated Polymers, Adv. Funct. Mater., 2001, 11(3), 229-234.

13 M. Abdelsamie, K. Zhao, M. R. Niazi, K. W. Chou and A. Amassian, In Situ UV-Visible Absorption during SpinCoating of Organic Semiconductors: A New Probe for Organic Electronics and Photovoltaics, J. Mater. Chem. C, 2014, 2(17), 3373-3381.

14 J. Gao, E. T. Niles and J. K. Grey, Aggregates Promote Efficient Charge Transfer Doping of Poly(3- Hexylthiophene), J. Phys. Chem. Lett., 2013, 4(17), 2953-2957.

15 B. Neelamraju, K. E. Watts, J. E. Pemberton and E. L. Ratcliff, Correlation of Coexistent Charge Transfer States in F4TCNQDoped P3HT with Microstructure, J. Phys. Chem. Lett., 2018, 9(23), 6871-6877.

16 I. E. Jacobs, C. Cendra, T. F. Harrelson, Z. I. Bedolla Valdez, R. Faller, A. Salleo and A. J. Moulé, Polymorphism Controls the Degree of Charge Transfer in a Molecularly Doped Semiconducting Polymer, Mater. Horiz., 2018, 5(4), 655-660.

17 F. M. McFarland, L. R. Bonnette, E. A. Acres and S. Guo, The Impact of Aggregation on the P-Doping Kinetics of Poly(3Hexylthiophene), J. Mater. Chem. C, 2017, 5(23), 5764-5771.

18 K. Tang, F. M. McFarland, S. Travis, J. Lim, J. D. Azoulay and S. Guo, Aggregation of P3HT as a Preferred Pathway for Its Chemical Doping with F4TCNQ, Chem. Commun., 2018, 54(84), 11925-11928.

19 F. M. McFarland, C. M. Ellis and S. Guo, The Aggregation of Poly(3-Hexylthiophene) into Nanowires: With and without Chemical Doping, J. Phys. Chem. C, 2017, 121(8), 4740-4746.

20 I. E. Jacobs, E. W. Aasen, J. L. Oliveira, T. N. Fonseca, J. D. Roehling, J. Li, G. Zhang, M. P. Augustine, M. Mascal and A. J. Moulé, Comparison of Solution-Mixed and Sequentially Processed P3HT:F4TCNQ Films: Effect of DopingInduced Aggregation on Film Morphology, J. Mater. Chem. C, 2016, 4(16), 3454-3466.

21 C. Wang, D. T. Duong, K. Vandewal, J. Rivnay and A. Salleo, Optical Measurement of Doping Efficiency in Poly(3Hexylthiophene) Solutions and Thin Films, Phys. Rev. B, 2015, 91(8), 085205.

22 A. R. Chew and A. Salleo, Spectroscopic Studies of DopantInduced Conformational Changes in Poly(3-Hexylthiophene) Thin Films, MRS Commun., 2017, 7(3), 728-734.
23 J. B. Torrance, J. J. Mayerle, K. Bechgaard, B. D. Silverman and Y. Tomkiewicz, Comparison of Two Isostructural Organic Compounds, One Metallic and the Other Insulating, Phys. Rev. B, 1980, 22(10), 4960-4965.

24 H. Méndez, G. Heimel, S. Winkler, J. Frisch, A. Opitz, K. Sauer, B. Wegner, M. Oehzelt, C. Röthel, S. Duhm, D. Többens, N. Koch and I. Salzmann, Charge-Transfer Crystallites as Molecular Electrical Dopants, Nat. Commun., 2015, 6(1), 8560.

25 G. C. Welch, R. Coffin, J. Peet and G. C. Bazan, Band Gap Control in Conjugated Oligomers via Lewis Acids, J. Am. Chem. Soc., 2009, 131(31), 10802-10803.

26 R. J. Kwaan, C. J. Harlan and J. R. Norton, Generation and Characterization of the Tris(Pentafluorophenyl)Borane Radical Anion, Organometallics, 2001, 20(17), 3818-3820.

27 B. Yurash, D. X. Cao, V. V. Brus, D. Leifert, M. Wang, A. Dixon, M. Seifrid, A. E. Mansour, D. Lungwitz, T. Liu, P. J. Santiago, K. R. Graham, N. Koch, G. C. Bazan and T.-Q. Nguyen, Towards Understanding the Doping Mechanism of Organic Semiconductors by Lewis Acids, Nat. Mater., 2019, 18(12), 1327-1334.

28 M. Roesing, J. Howell and D. Boucher, Solubility Characteristics of Poly(3-Hexylthiophene), J. Polym. Sci., Part B: Polym. Phys., 2017, 55(14), 1075-1087.

29 F. Machui, S. Langner, X. Zhu, S. Abbott and C. J. Brabec, Determination of the P3HT:PCBM Solubility Parameters via a Binary Solvent Gradient Method: Impact of Solubility on the Photovoltaic Performance, Sol. Energy Mater. Sol. Cells, 2012, 100, 138-146.

30 P. J. Brown, D. S. Thomas, A. Köhler, J. S. Wilson, J.-S. Kim, C. M. Ramsdale, H. Sirringhaus and R. H. Friend, Effect of Interchain Interactions on the Absorption and Emission of Poly(3-Hexylthiophene), Phys. Rev. B, 2003, 67(6), 064203.

31 S. G. Urquhart, M. Martinson, S. Eger, V. Murcia, H. Ade and B. A. Collins, Connecting Molecular Conformation to Aggregation in P3HT Using Near Edge X-Ray Absorption Fine Structure Spectroscopy, J. Phys. Chem. C, 2017, 121(39), 21720-21728.

32 J. Clark, C. Silva, R. H. Friend and F. C. Spano, Role of Intermolecular Coupling in the Photophysics of Disordered Organic Semiconductors: Aggregate Emission in Regioregular Polythiophene, Phys. Rev. Lett., 2007, 98(20), 206406.

33 T. J. Prosa, M. J. Winokur, J. Moulton, P. Smith and A. J. Heeger, X-Ray Structural Studies of Poly(3-Alkylthiophenes): An Example of an Inverse Comb, Macromolecules, 1992, 25(17), 4364-4372.

34 D. T. Duong, M. F. Toney and A. Salleo, Role of Confinement and Aggregation in Charge Transport in Semicrystalline Polythiophene Thin Films, Phys. Rev. B, 2012, 86(20), 205205.

35 R. Noriega, J. Rivnay, K. Vandewal, F. P. V. Koch, N. Stingelin, P. Smith, M. F. Toney and A. Salleo, A General Relationship between Disorder, Aggregation and Charge Transport in Conjugated Polymers, Nat. Mater., 2013, 12(11), 1038-1044.

36 E. L. Ratcliff, J. L. Jenkins, K. Nebesny and N. R. Armstrong, Electrodeposited, “Textured” Poly(3-Hexyl-Thiophene) (e-P3HT) Films for Photovoltaic Applications, Chem. Mater., 2008, 20(18), 5796-5806. 
37 R. C. Shallcross, T. Stubhan, E. L. Ratcliff, A. Kahn, C. J. Brabec and N. R. Armstrong, Quantifying the Extent of Contact Doping at the Interface between High Work Function Electrical Contacts and Poly(3-Hexylthiophene) (P3HT), J. Phys. Chem. Lett., 2015, 6(8), 1303-1309.

38 S. Hotta, S. D. D. V. Rughooputh, A. J. Heeger and F. Wudl, Spectroscopic Studies of Soluble Poly(3-Alkylthienylenes), Macromolecules, 1987, 20(1), 212-215.

39 V. M. Geskin and J. L. Brédas, Polaron Pair versus Bipolaron on Oligothiophene Chains: A Theoretical Study of the Singlet and Triplet States, ChemPhysChem, 2003, 4(5), 498-505.

40 J. Yamamoto and Y. Furukawa, Electronic and Vibrational Spectra of Positive Polarons and Bipolarons in Regioregular Poly(3-Hexylthiophene) Doped with Ferric Chloride, J. Phys. Chem. B, 2015, 119(13), 4788-4794.

41 K. Tashiro, K. Ono, Y. Minagawa, K. Kobayashi, T. Kawai and K. Yoshino, Structural Changes in the Thermochromic Solid-State Phase Transition of Poly(3-Alkylthiophene), Synth. Met., 1991, 41(1-2), 571-574.

42 K. Rahimi, I. Botiz, N. Stingelin, N. Kayunkid, M. Sommer, F. P. V. Koch, H. Nguyen, O. Coulembier, P. Dubois, M. Brinkmann and G. Reiter, Controllable Processes for
Generating Large Single Crystals of Poly(3-Hexylthiophene), Angew. Chem., Int. Ed., 2012, 51(44), 11131-11135.

43 A. Bohle, D. Dudenko, N. Koenen, D. Sebastiani, S. Allard, U. Scherf, H. W. Spiess and M. R. Hansen, A Generalized Packing Model for Bulk Crystalline Regioregular Poly(3Alkylthiophenes) with Extended Side Chains, Macromol. Chem. Phys., 2018, 219(3), 1700266.

44 H. Hase, K. O’Neill, J. Frisch, A. Opitz, N. Koch and I. Salzmann, Unraveling the Microstructure of Molecularly Doped Poly(3-Hexylthiophene) by Thermally Induced Dedoping, J. Phys. Chem. C, 2018, 122(45), 25893-25899.

45 D. Beljonne, J. Cornil, R. Silbey, P. Millié and J. L. Brédas, Interchain Interactions in Conjugated Materials: The Exciton Model versus the Supermolecular Approach, J. Chem. Phys., 2000, 112(10), 4749-4758.

46 M. Muccini, E. Lunedei, C. Taliani, D. Beljonne, J. Cornil and J. L. Brédas, Interchain Interaction in a Prototypical Conjugated Oligomer from Polarized Absorption at $4.2 \mathrm{~K}$ : $\alpha$-Sexithienyl Single Crystal, J. Chem. Phys., 1998, 109(23), 10513-10520.

47 P. Petelenz and M. Andrzejak, Davydov Splitting in the Sexithiophene Crystal, Chem. Phys. Lett., 2001, 343(1-2), 139-142. 